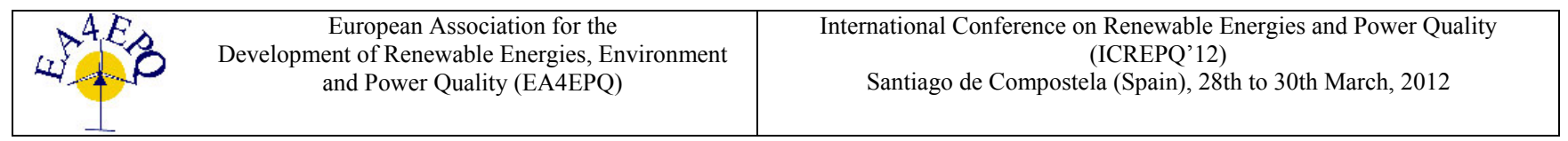

\title{
Determining Optimal Breakpoints in Urban Power Networks with Genetic Algorithm
}

\author{
S.E.Kokin ${ }^{1}$, S.A.Dmitriev ${ }^{2}$ and A.I.Halyasmaa ${ }^{3}$ \\ ${ }^{1}$ Department of Automated Electric Systems \\ Ural Energy Institute \\ Ural Federal University named after $1^{\text {st }}$ Russian president B.N.Yeltsin \\ 19 Mira street, Yekaterinburg, Russia 620002 \\ Phone/Fax number:+7 34335916 15, Mobile +7 9122419 376, e-mail: kokinser@list.ru \\ ${ }^{2}$ Department of Automated Electric Systems \\ Ural Energy Institute \\ Ural Federal University named after $1^{\text {st }}$ Russian president B.N.Yeltsin \\ 19 Mira street, Yekaterinburg, Russia 620002 \\ Phone/Fax number:+7 34335916 15, email: dmstepan@gmail.com \\ ${ }^{3}$ Department of Automated Electric Systems \\ Ural Energy Institute \\ Ural Federal University named after $1^{\text {st }}$ Russian president B.N.Yeltsin \\ 19 Mira street, Yekaterinburg, Russia 620002 \\ Phone/Fax number:+7 34335916 15, email: 1khalyasmaa@mail.ru
}

\begin{abstract}
The article deals with the issues of optimizing the operating modes of $6-10 \mathrm{kV}$ distribution networks constituted by the large-sized complicated meshed systems. In real time, such networks operate with breaking the circuit to eliminate equalizing currents. The goal of optimizing the networks' operating modes is to minimize the active power loss. Determining the optimal breakpoints is a complicated discrete task, for which the method of genetic algorithm becomes the most suitable solution.
\end{abstract}

\section{Key words}

Power Supply System, Optimization, Genetic Algorithm.

\section{Introduction}

Urban power supply systems aim to provide electric power for technological processes of all utility, production, transportation and other consumers that are situated on their territories. Big cities with modern and rational electric networks are marked (with the exception of general purpose networks below $1 \mathrm{kV}$ ) by combination of various voltage classes ranging from $6 \mathrm{kV}$ to $500 \mathrm{kV}$.

Traditionally $10(6) \mathrm{kV}$ distribution networks (DN) are the weakest points of the system. Such networks are characterized by large size (tens and hundreds thousands of main substations). The complex load structure requires networks with power back-up. The complex closed topology of the units complicates the analysis of the power transportation processes in the $10(6) \mathrm{kV}$ DNs. Looped and bulk power systems in normal regime operate with breaking the circuit in one of the units to eliminate equalizing currents in closed circuits which generate extra power loss. Breaking the circuit (determining the optimal breakpoints) is an important operational task, solving which will contribute to reliability and quality of power supply of the consumers and will allow for reducing power loss during transmission.

\section{Devising Genetic Algorithm for Determining Optimal Breakpoints.}

We shall apply Genetic algorithm as a base method for determining optimal breakpoints of closed circuits of the urban power network [1]. In a simplified form, Genetic Algorithm can be described as follows:

Step 1. Formation of the initial population. The population is formed by the method of random generation in the range of the possible values, i.e. the number of chromosomes in population and the number of genes in a chromosome is set in the program.

Step 2. Calculate the fitness of each chromosome of the initial population equal to the target function value, i.e. to the active power loss. The resulted regime of the power 
network is calculated by the Newton method for each chromosome in each generation.

The software using the genetic algorithm addresses the computation complex of the resulted regime to calculate the target function values, i.e. the total active power loss. A chromosome is represented by 1 and 0 values, where the zeroes stand for the break in circuit. Shall the program meet a zero in a chromosome, it will eliminate the index L before the corresponding line in the field of the initial data NET, and start the computation of the regime, at the same time memorizing and storing in the database the power loss values. When the solution is not possible or the required preciseness of the last iterations cannot be obtained (in total 10 iterations are used), the so-called penalty function is applied. Penalty function eliminates the chromosome from the population but stores the information on this chromosome in the database.

Step 3. Verifying the Stop Condition of the algorithm.

The Stop Criteria of the algorithms represents the situation when further continuous application of the algorithm does not lead to further improvement of the already reached value. The algorithm may also be stopped after producing the specific number of generations. When the Stop Criteria is fulfilled, the program goes to the final stage of selecting the "best fit" chromosome. Otherwise, the selection is carried out at the next step.

Step 4. Life cycle of the population is represented by a number of genetic operators:

- Selection of the parent couple. Chromosomes to participate in formation of the new population are selected by applying the "roulette method" operator;

- Crossover of the selected chromosomes to get new solutions. For physical variables with probability $P_{c r}$ an arithmetic crossover is applied;

- Mutation of one randomly selected gene in each chromosome with the pre-set probability $P_{m u t}$ in accordance with the uniform mutation operator. The gene at hand assumes a new value that is randomly selected from the allowance range.

Step 5. Formation of the new population. The chromosomes obtained as the result of application of the genetic operators to the chromosomes of the temporary parent population are included in the new population which becomes the so-called current population for the present iteration of the genetic algorithm. After completion of Step 5, go to Step 2.

Step 6. Selection of the "best fit" chromosome. If the Stop Condition of the algorithm is fulfilled, the result, i.e. the solution for the problem at hand, is presented.

The devised genetic algorithm of determination of the optimal breakpoints of the electric network was implemented in the integrated development environment of the Delphi software.

\section{Implementing Genetic Algorithm to determine breakpoints in distribution network.}

Effectiveness of the suggested genetic algorithm and efficacy of the software was proved by calculations of real power flow of the part of electric chain in the city of Yekaterinburg, Russia.

The number of chromosomes means the number of chromosomes in the population, it is randomly set, and the chromosomes are generated randomly each time. The more chromosomes there is in the population, the less number of generations it will take to find the optimal solution.

After that, the probability of the main genetic operators of the classical genetic algorithm is set: crossover operator and mutation operator. In the classical genetic algorithm, crossover is applied nearly every time, while the mutation is applied rarely enough. The probability of crossover is quite high $\left(0,5<P_{c r}<1\right)$, while the probability of mutation is set quite low $\left(0<P_{m u t}<0,1\right)$. It is important to keep within this range, or otherwise the obtained result will not be valid.

The operator of inversion is an additional operator applied to get new offsprings. Inversion is applied to the single chromosome, and the application of inversion changes the consequence of genes in the two randomly selected points of the chromosome. This additional operator is not frequently used in the computational practice of the genetic algorithms. Application of this operator allows to obtain close to optimal solution in fewer number of populations, thus reducing the time necessary for the calculations.

Changing the probability values will affect the number of populations used during the computation.

Also, the Stop Condition of the program may vary. In our case, the Stop Condition was set as the number of the runs during which the fitness function (target function) does not change its value. Increasing the number of the runs will lead to decreasing the value of the target functions, and therefore will allow to obtain the solution that will correspond to the minimum power loss in the network.

Before starting the computation, let us analyze the scheme to determine the number of possible breakpoints in the network. For the network in question, there will be 16 possible breakpoints.

As a result, we obtained the target function value that corresponded to the minimum power loss and the "best fit" chromosome. Such chromosome encodes the breakpoints of the network in question. The value 1 stands for the absence of the break in the unit; the value 0 stands for the presence of the break in the unit. The solution obtained during the computation is presented in Figure 1. 


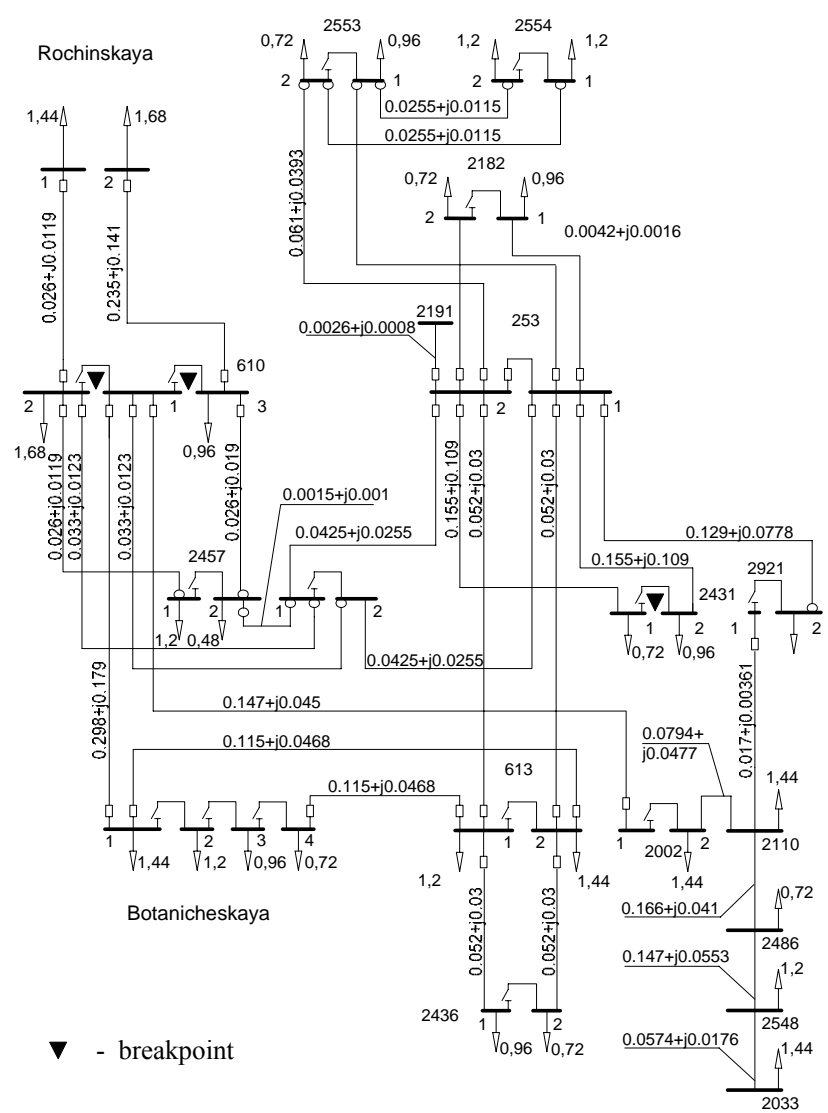

Fig. 1. Graphic presentation of the solution obtained by GA.

Active power loss in the obtained scheme is lower than in the closed variant, but one cannot say for sure that it was the optimal variant as genetic algorithm method does not belong to precise computational methods. What we can say is that we found a good enough solution compared to the closed circuit scheme we started our reasoning from.

The active power loss $\Delta P$ decreased to $151,8 \mathrm{~kW}$. To obtain even bigger loss reduction it is necessary either to expand the initial population or to stretch the stopping criteria of the algorithm thus increasing the number of variants considered while looking for optimal solution. In other words, reduction of power loss can be reached by changing the parameters of the probability of application of the main operators of the GA.

It is worth notable that with the small size of population and the Stop Criteria less than 1000, the solution may be obtained by adjusting the probability of application of the main operators. The target function value of such solution will be higher than that of the solution obtained with application of the operators with lower probability. It is explained by the fact that the chromosomes in the initial population are created randomly, and the algorithm does not manage to try all possible gene combinations.

\section{Conclusion.}

Effectiveness of the suggested genetic algorithm is proved by computation of the regime and comparison of active power loss before and after the application of the said genetic algorithm. In the initial closed scheme, the power loss was $210 \mathrm{~kW}$, while after the application of the developed genetic algorithm and determining the optimal breakpoints the active power loss was reduced by $27,7 \%$ to $151,8 \mathrm{~kW}$.

Therefore it is possible to conclude that genetic algorithm allows to find optimal solution and does not contain any requirements for continuity and differentiability of the target function, because it does not require derivations, and provides for the search of the global minimum when the task contains several minima.

\section{References}

[1] D. E. Goldberg, Genetic Algorithms in Search, Optimization and Machine Learning, Reading, MA: Addison-Wesley (1989).

[2] Simon Haykin, Neural Networks and Learning Machines (3rd Edition) (2008).

[3] Christopher M. Bishop, Neural Networks for Pattern Recognition (1996).

[4] Kevin Gurne, An Introduction to Neural Networks (1997).

[5] V. V. Kruglov, V. V. Borisov, Artificial Neural Networks. Theory and practice. - Moscow.: Hot line - Telecom, 2001. 382. (2000). 